$$
\text { DOE/PC/93210-9 }
$$

\title{
Radiation/Turbulence Interactions
}

$$
\text { in Pulverized-Coal Flames meotred }
$$

Aบก 25 1996

OSTI

M. P. MENGÜÇ, J. M. McDONOUGH,

S. MANICKAVSAGAM, S. MUKERJI, and S. SWABB

\author{
Department of Mechanical Engineering \\ UNIVERSITY OF KENTUCKY \\ LEXINGTON, KY 40506
}

Technical Progress Report: Third Year, Second Quarter Period: December 15, 1995 - March 15, 1996

No: $\mathrm{DOE} / \mathrm{PC} / 93210-9$

(Grant \#DE-FG22-93PC93210)

\author{
submitted to \\ DEPARTMENT OF ENERGY, \\ Pittsburgh Energy Technology Center, \\ Pittsburgh, Pennsylvania
}

DSTTIEUTION OF THIS DOCUMENT IS UNLEGTEO 


\section{DISCLAIMER}

Portions of this document may be illegible in electronic image products. Images are produced from the best available original document. 


\section{DISCLAIMER}

This report was prepared as an account of work sponsored by an agency of the United States Government. Neither the United States Government nor any agency thereof, nor any of their employees, makes any warranty, express or implied, or assumes any legal liability or responsibility for the accuracy, completeness, or usefulness of any information, apparatus, product, or process disclosed, or represents that its use would not infringe privately owned rights. Reference herein to any specific commercial product, process, or service by trade name, trademark, manufacturer, or otherwise does not necessarily constitute or imply its endorsement, recommendation, or favoring by the United States Government or any agency thereof. The views and opinions of authors expressed herein do not necessarily state or reflect those of the United States Government or any agency thereof. 


\section{Preface}

This is the second quarter Technical Report for the third year of the project titled "Radiation-Turbulence Interactions in Pulverized-Coal Flames", which is funded by the Department of Energy, Pittsburgh Energy Technology Center (Grant \#DE-FG22-93PC93210). The report covers the period from December 15, 1995 to March 15, 1996.

One research associate and two Ph.D. students are involved in this phase of the project. Additional support for these researchers has been received from the Department of Mechanical Engineering at UK.

Dr. Sivakumar Manickavasagam, a Research Associate, is primarily responsible for combining the fluid mechanics and radiative transfer computer codes. He is also in charge of developing a new algorithm to account for the soot agglomerate structure and char fragmentation. He was also responsible in running the experiments. Mr. Sudip Mukerji, a Ph.D. student, is developing the computer codes for simulation of turbulence using the additive turbulent decomposition (ATD) technique in two and three dimensional geometries. Mr. Derong Wang, another Ph.D. student is primarily supported by a NSF/EPSCoR project. He is modeling the interaction of radiation beam with small scale ATD technique. Mr. Steve Swabb conducted extensive experiments with and without coal particles to determine the chaotic signals.

During this period, we have completed a paper, which was submitted for presentation at the International Mechanical Engineering Congress and Exposition, which will be held in Atlanta, November 1996. This report is based on this paper, titled: FLUCTUATIONS OF SOOT IN TURBULENT DIFFUSION FLAMES: EXPERIMENTAL DATA AND CHAOTIC MAP MODELS. 


\title{
FLUCTUATIONS OF SOOT IN TURBULENT DIFFUSION FLAMES: EXPERIMENTAL DATA AND CHAOTIC MAP MODELS
}

\author{
M.P. MENGÜÇ, J.M. McDONOUGH, S. MANICKAVASAGAM, S. MUKERJI, S. \\ SWABB, S. GHOSAL \\ Department of Mechanical Engineering, \\ University of Kentucky, \\ Lexington, KY 40506-0046, USA
}

\begin{abstract}
:
In this paper, we discuss an experimental and theoretical methodology to characterize soot volume fraction fluctuations in turbulent diffusion flames via chaotic maps. The approach is based on the hypothesis that the fluctuations of properties in turbulent flames is deterministic in nature, rather than statistical. To this extent, we measured the time series of soot scattering coefficient in an ethylene diffusion flame from light scattering experiments. Following this, corresponding power spectra and delay maps were calculated. It was shown that if the data were averaged, the characteristics of the fluctuations were almost completely washed out. The psds from experiments were successfully modeled using a series of logistic maps.
\end{abstract}

\section{INTRODUCTION}

In combustion systems, several complicated phenomena occur simultaneously, including turbulent fluid flow, heat transfer, radiation transfer, chemical kinetics, phase change leading to pollution formation, etc. The nature of each one of these physical mechanisms is very complicated and more importantly, depends on others. Yet, most of the studies performed over the years treated each one of these mechanisms independently, and did not consider the interactions between them in detail. Among all these phenomena, probably radiation transfer and turbulence deserve special recognition for flame studies. However, turbulent 
fluid flow is still not very well understood, and radiation transfer is too complicated to be modeled in detail. Even if it is modeled, the accuracy of predictions is questionable unless the medium properties are available. Ironically, these properties, e.g. soot agglomerate size, structure and volume fraction distribution, cannot be predicted with confidence without modeling the interaction of chemical kinetics, turbulence and radiation transfer!

Detailed discussion of radiation-turbulence interactions in flames is available in the literature (see, e.g., [1-6]). Despite all these studies and the importance of radiation-turbulence interactions throughout the field of combustion, the problem remains "unsolved" in the sense that it is currently not possible to predict flow and heat transfer in high-Reynolds number (Re) combusting systems of practical importance with any degree of confidence. It must first be admitted that modeling of turbulent flows, even in the absence of interactions, has generally been unsuccessful in the context of the Reynolds-averaged Navier-Stokes (N.-S.) equations as has been recently demonstrated by Freitas [7]. If progress is to be made, it will probably have to be in directions different from past efforts. In particular, it is simply not possible to correctly account for time-dependent interactions by using equations that merely model statistics, even if these equations are highly accurate.

This is easily seen by considering prediction of behavior of a flame in an intermittent, gusty flow field. Statistical analysis would be likely to predict preservation of a steady combustion process at some level, whereas the physics of the situation would dictate that sooner or later the flame would be extinguished by a gust, and thereafter there would be no combustion. To a somewhat lesser extent, this same difficulty arises also in large-eddy simulation (LES). In light of the obvious importance of understanding, and thus being able to control, combustion processes, and the fact that past radiation-turbulence interaction analyses have not been particularly successful, we have begun studies that represent a distinct divergence from the earlier modeling techniques.

In this paper we will present a series of experiments and a novel modeling approach for accounting for interactions of turbulence with radiation. This technique was formally introduced by Hylin and McDonough [8] in the context of an overall turbulence modeling procedure known as additive turbulent decomposition (ATD), but it had been employed previously in a somewhat ad hoc way by, e.g., Sreenivasan and Ramshankar [9]. The overall ATD procedure consists of solving large-scale, unaveraged governing equations containing small-scale dependent variables that are directly modeled in some manner. It is important to recognize that this represents a significant departure from the usual Reynolds-averaged methods in which it is the statistics, i.e., correlations, of dependent variables that are modeled. It is this feature that leads to the ability to directly model interactions of turbulence with other phenomena, but at the same time it raises new issues regarding construction of the required models. Methods for carrying out such constructions, and results obtained in a specific case, namely turbulent, radiating flow in a diffusion flame, will be the subject of 
this paper.

The key underlying idea is to employ one-dimensional chaotic maps of the unit interval as the foundation for the model [10]. The rationale behind this stems from the "universality" properties of such maps, as demonstrated by Feigenbaum [11], and others, and particularly from the fact that their bifurcation sequences can be similar to those of the N.-S. equations (see [12]). Thus, they appear to provide good candidates for models of fluctuating variables associated with fluid flow phenomena.

Here, we focus only on the use of chaotic maps for quantification of soot mass fluctuations in turbulent diffusion flames. Soot volume fraction plays a major role in radiation-turbulence interactions in flames. In general, soot formation and oxidation processes are controlled by the chemical kinetics, which is a strong function of temperature and species gradients. Both of these gradients are affected by the structure of the turbulent flow field and strong radiative heating and/or cooling. It will be shown that the radiation-turbulence interactions in sooting diffusion flames can be deterministic in nature. The present work is the first to demonstrate that experimental data can be used in a direct and straightforward way to construct chaotic algebraic maps to quantify these interactions.

Radiant energy emitted from a sooty flame is significantly greater than, for example, that from a clean burning natural gas flame. In order to predict the contribution of soot to overall radiative transfer phenomena in combustion chambers, one must know the soot shape, size, size distribution, optical properties and volume fraction distribution. Recently, Manickavasagam and Mengüç [13] reviewed the scattering characteristics of soot agglomerates and presented a new methodology to determine soot size and structure from ellipticallypolarized light scattering experiments. This information is crucial for identification of soot volume fraction distribution from the data collected from light scattering experiments.

\section{EXPERIMENTS}

\subsection{Experimental System}

The underlying intent of the experiments conducted for this project is to obtain data on angularly scattered light intensity as a function of time (referred to as time series). This variation in recorded intensity is related to transience of the scattering particles (soot) in the control volume. A diagnostic system based on a short pulse, solid state Nd:YAG laser and on an optical parametric oscillator (OPO) has been developed (see Fig. 1).

The $532 \mathrm{~nm}$ wavelength (green) of the $\mathrm{Nd}$ YAG laser is used in the experiments. This Q-switched laser produced pulsed radiation at $30 \mathrm{~Hz}$ with a very short pulse width of 4$9 \mathrm{~ns}$. The laser beam is focussed on to the flame and has a diameter of about $2 \mathrm{~mm}$ at the 
measurement plane. Two nine-stage, side-on PMTs (a RCA 4840 and a Hamamatsu R446), with a detection range of $200-900 \mathrm{~nm}$, measure the scattered light and the reference signal simultaneously.

The SRS 250 gated-integrator and boxcar averager module are employed to recover analog signals. The gate generator, which can be triggered externally (e.g., by the laser's Q-switch Synch output signal), provides an adjustable delay in the range of $1 \mathrm{~ns}-10 \mathrm{~ms}$, and generates a gate of continuously variable width in the range $2 \mathrm{~ns}-15 \mu \mathrm{s}$. Only that part of the signal overlapping with the gate is recorded. A dual-channel $400 \mathrm{MHz}$ oscilloscope (HP 54502A) is used to adjust the delay so that the gate is positioned over the signal.

The fast-gated integrator integrates the input signal, its output being normalized by the gate width to produce a (further amplifiable) voltage proportional to the average input signal during the sampling gate. The signal can be monitored using analog or digital meters on the SRS 280 System Mainframe. The Computer Interface Module (SRS 245) is used to transfer the data to a PC where a commercial data acquisition software (Labtech) receives, plots, and records the data. Two gated integrators are being used to acquire both the scattered signal and the reference signal simultaneously.

Experiments were conducted using a coflow diffusion flame burner. The overall height of the burner assembly is $35 \mathrm{~cm}$. It is mounted on a mechanism enabling vertical and horizontal translations of about 15 and $5 \mathrm{~cm}$, respectively. The burner consists of a central fuel tube surrounded by a co-annular oxidizer tube having diameters of 1 and $5 \mathrm{~cm}$, and has been designed to produce a flat velocity profile of the oxidizer and the fuel. The oxidizer tube has two diametrically opposite inlet ports which make possible an even distribution of the oxidizer across the cross-section of the tube. An $8 \mathrm{~cm}$ deep layer of $4 \mathrm{~mm}$ diameter glass beads within the conical portion of the oxidizer passage and four thin metallic sheets having (uniformly distributed) $3 \mathrm{~mm}$ diameter holes placed at constant intervals across the cylindrical portion of the oxidizer passage ensure a stable and radially-uniform flow of the oxidizer. Ethylene (99.5\% purity) was used as fuel. Line air supply to the laboratory, filtered and precisely regulated, was used as oxidizer.

\subsection{Experimental Results}

Experiments were conducted to obtain data on scattered light intensity from soot particles at $30^{\circ}$, providing a measure of soot volume fraction, $f_{v}$, as a function of time. The time series were obtained by making single-shot measurements at the rate of thirty data points per second (i.e., at laser pulse frequency) for an ethylene flame using several different air-fuel ratios, and measurements were recorded at several different heights.

The most fundamental hypothesis in our approach to analyzing these data, and subsequently 
constructing turbulence models, is that turbulent fluid flows and any associated interacting phenomena are deterministic, rather than stochastic, in nature. It should be noted that this hypothesis is amply supported by both theoretical and experimental results beginning with the work of Ruelle and Takens [14]. It is therefore reasonable to employ deterministic dynamical systems as turbulence models, and we remark that the N.-S. equations comprise one such system. But there exist much simpler systems possessing many of the features of the N.-S. equations, viz., chaotic time series and bifurcation sequences leading from steady to periodic, and on to chaotic behavior.

There are a number of important tools that have been developed for the qualitative and quantitative analysis of dynamical systems, or more precisely, for analysis of time series generated by the evolution of such systems. The reader is referred to References [15] and [16] for very readable treatments. Here, we will specifically employ the following in the analysis of our experimental data: i) time series, ii) power spectral density (psd), and iii) delay maps; but we caution that use of additional ones such as Poincaré maps, correlation dimension, etc. may be necessary to obtain more refined results. Moreover, it should be emphasized that none of these quantities, psd, etc., can be inverted to imply a unique time series. Thus the modeling effort must employ more sophisticated analyses and quantification of the time series behavior itself, e.g., use of total variation per unit time, frequency of zero crossings, etc, in order to more precisely model complex, aperiodic deterministic phenomena.

We begin with direct observation of time series of measured data in an effort to detect qualitative features of the temporal behavior of the phenomenon under consideration such as periodicity, intermittency or more complex behavior. Comparison of time series from different spatial locations and/or for different values of a control (bifurcation) parameter, e.g., air-fuel ratio, may reveal features suggesting a particular bifurcation sequence, and thus a particular chaotic map (or maps) to be employed as a model.

Figure 2 depicts the composite picture of the flame and corresponding time series, psd and delay maps at four axial locations. The time series suggest that the flow becomes more turbulent at higher axial locations (increasing $R e$ ). In particular, these data are strongly suggestive of a subharmonic bifurcation sequence of the Feigenbaum type (see [11]).

Following this we calculate the psd of the time series to further elucidate any details of periodic, quasi-periodic, subharmonic or chaotic behavior. We comment that for turbulent, chaotic data it is typical for the psd to consist of a broad-band spectrum (significant power over a wide range of frequencies) with $f^{-\alpha}(0<\alpha<2)$ decay. The psds displayed in Fig. 2 show very little of this typical $f^{-\alpha}$ decay, but on the other hand there is clear evidence of a subharmonic bifurcation sequence, that is well underway already at the lowest measurement location.

The final diagnostic technique employed in the present study is construction of delay maps. 
These can be viewed as phase diagrams, but ones constructed from the time series of a single variable, say $f_{v}$, by shifting the values of $f_{v}$ uniformly by $\Delta t$ (the delay), and plotting $f_{v}(t)$ vs. $f_{v}(t-\Delta t)$. Delay maps exhibit features quite analogous to those of phase portraits. In particular, periodic behavior is represented by a single closed loop (limit cycle); subharmonic behavior appears also as a closed loop, but one which intersects itself a certain number of times depending on the degree of subharmonicity (i.e., period doubled, tripled, etc.). The delay map of a chaotic time series associated with a strange attractor is typically quite complex, but it is readily distinguished from one arising from a random process in that the latter is "space filling" while the former is not. That is, the trajectory of a (normalized) random process will visit every point in the unit square, but that of a strange attractor associated with a deterministic dynamical system will pass through only some fraction (strictly less than unity, and typically quite small) of the points. This distinct difference provides a fairly reliable indicator, albeit a qualitative one, of chaotic dynamics. The results shown in Figure 2 strongly suggest deterministic origin of the observed behavior. Moreover, it appears that although there are differences in detail, the topology of the maps is similar providing further indication that the chaos has probably arisen from a subharmonic sequence.

As it was discussed before, the most novel part of this new approach is the fact that every bit of the data is used to understand the underlying physics. In most studies, the results are based on averaged data, which filters out many characteristics of a given physical phenomenon. In order to show the adverse effect of averaging, we carried out the experiments for the same flame twice, but collected the data using two different approaches. In the first case, we recorded every scattered laser light received by the detector. This means that thirty signals were recorded every second. For the next case, we recorded the average of thirty data points; in other words, one data point is recorded every second. Both experiments were carried out for 15 minutes; corresponding psds are displayed in Fig. 3. It is obvious that when the data are averaged, psds show no evidence of a subharmonic bifurcation sequence. Indeed, the psds corresponding to two different heights in the flame are indistinguishable, whereas for the unaveraged case, the psds show increasing chaotic behavior with increasing axial location in the flame (or, increasing $R e$ ). Comparisons of the time series for these cases reveal that the averaging does not affect the low-frequency fluctuations, as expected; however, almost all the details of the high frequency fluctuations are lost. These high frequency fluctuations are believed to be produced due to the shear flow oscillations, which are initiated because of the different flow rates of air and fuel in the co-flow burner. Experimental error is out of question, as these oscillations go through a bifurcation sequence with increasing height in the flame. Data acquisition error would show up in similar structure at all heights.

This figure is very important for demonstrating that if detailed understanding of turbulence and its interaction with radiation and/or chemical kinetics are to be studied (and modeled), one cannot rely on the averaged data. In particular, the basic type of bifurcation sequence, the knowledge of which is critical to our modeling approach, cannot be discerned from 
averaged data.

\section{MODEL CONSTRUCTION}

We begin model construction by noting that the power spectra presented in Fig. 2 are very similar to those obtained from the time series of the logistic map, a widely studied nonlinear algebraic map (cf. [10]). Such strong similarities earlier motivated us to employ a slightly modified form of the logistic map,

$$
x^{n+1}=b x^{n}\left(1-C\left|x^{n}\right|\right)
$$

as a simple model for fluctuating absorptivity [6]. Here, $b$ is the bifurcation parameter (analogous to $R e$ in the N.-S. equations), and $C$ is a normalization constant. Equation (1) has also been used in McDonough et al. [17] as a model for fluctuating small-scale velocity in turbulent backward-facing step flows. Although there are strong similarities between the experimental results and those obtained from the logistic map, there are also obvious differences in detail. For the present experimental data the psds clearly show at least two distinct time scales in the spatially lower part of the flame, and a simple logistic map such as Eq. (1) is not able to replicate this. Moreover, these same data indicate a large number of harmonics of the fundamental frequency, and these cannot be produced by a single logistic map.

There are, however, numerous means by which these shortcomings might be addressed. Here we will employ a simple linear combination of logistic maps of the form (1):

$$
x^{n+1}=\sum_{k=1}^{K} \alpha_{k} x_{k}^{n+1},
$$

where each $x_{k}$ is calculated from Eq. (1), but possibly with different values of $b$, and different frequencies of evaluation for each $k$. Figure 4 displays modeled results obtained in this fashion using $\alpha_{k}, b_{k}$ and frequency of evaluation, $\omega_{k}$. Comparison of this with Fig. 2 corresponding to experimental data indicates that the experimental results have been quite successfully modeled via this chaotic map approach, and one should therefore expect to correctly model detailed time-dependent radiation-turbulence interactions with the overall ATD/chaotic map modeling procedure presented by McDonough et al. [6].

It is important to emphasize that the time series produced by our model lack some of the details seen in the experimental data despite their accurate replication of psds and delay maps. We remark that this is a consequence of the fact that many different time series can lead to the same (or similar) psd or delay map, underscoring the need to develop additional techniques for time series analysis in this modeling context. 


\section{SUMMARY AND CONCLUSIONS}

In this paper we have introduced a novel approach to investigate the radiation-turbulence interactions in diffusion flames. We have presented experimental data from a soot-laden turbulent diffusion flame produced with a co-flow burner and provided analyses of these data that indicate deterministic origin of soot volume fraction fluctuations. In particular, the data show strong evidence of a subharmonic bifurcation sequence from periodic behavior at the tip of the burner to fully turbulent at some height (depending on air/flow ratio) above the burner. On the basis of these observations we have constructed chaotic map models of this behavior using linear combinations of a modified logistic map.

We can draw several novel conclusions from this study:

- The radiation-turbulence interaction phenomenon in a soot-laden flame is evidently deterministic, rather than random, as has been shown previously for different flames by numerous investigators.

- It is possible to device fairly simple algebraic models of this quite complicated flow situation, but unlike earlier attempts with other physical systems, our model displays correct bifurcation sequences in addition to accurate behavior at individual parameter settings within the sequence. This implies a potential for "universality" and indicates that such models should be extremely useful in the context of the ATD/chaotic map turbulence modeling formalism.

- If the experimental data are averaged, either electronically during the data acquisition process, or later numerically, many fine physical characteristics of the system being studied are lost. This is a very important observation: in order to understand and study radiation-turbulence interactions accurately, we need time-resolved data for temperature, soot volume fraction, and species concentration distributions.

Acknowledgements: This work is supported by the DOE-PETC Advanced University Coal Research Program Grant No: DE-FG22-93PC93210. 


\section{REFERENCES}

1. Faeth, G.M., Jeng, S.M., and Gore, J., in Heat Transfer in Fire and Combustion Systems, (C.K. Law et al., Eds.), ASME, New York, 1985, HTD-Vol.45, pp.137-151.

2. Grosshandler, W.L., and Joulain, P., in Prog. Astro. and Aero., Vol. 105, Part II, AIAA, Washington, 1986, pp. 123-152.

3. Gore J.P., and Faeth G.M., ASME J. Heat Trans., 110:173-181 (1988).

4. Faeth, G.M., Gore J.P., Chuech, S.G. and Jeng, S.M., in Ann. Rev. Numerical Fluid Mech. and Heat Transfer, (C.L. Tien and T.C. Chawla, Eds.), Hemisphere, New York, 1989 , pp. 1-38.

5. Kounalakis, M.E., Sivathanu, Y.R. and Faeth, G.M., ASME J. Heat Transfer. 113:437445 (1991).

6. McDonough, J. M., Wang, D., and Mengüç, M.P., in Radiative Transfer - I : Proceedings of the First International Symposium on Radiative Transfer, (M.P. Mengüc, Ed.), Begell House, New York, 1996, (in press).

7. Freitas, C.J., J. Fluids Engineering, 117:208-218 (1995).

8. Hylin, E. C. and McDonough, J. M.,, University of Kentucky, Mech. Engr. Report, CFD-01-94, 1994.

9. Sreenivisan, K.R. and Ramshankar, R., Physica 23D:246-258 (1986).

10. Collet, P. and Eckmann, J.-P., Iterated Maps on the Interval as Dynamical Systems, Birkhäuser-Boston, Boston, 1980.

11. Feigenbaum, M.J., J. Stat. Phys., 19:25-52 (1978).

12. Pulliam, T.H. and Vastano, J.A., J. Comput. Phys, 105:133-149 (1993).

13. Manickavasagam, S. and Mengüç, M.P., Applied Optics, (1996) (in press).

14. Ruelle, D. and Takens, F., in Commun. Math. Phys., 20:167-192 (1971).

15. Guckenheimer, J. and Holmes, P., Nonlinear Oscillations, Dynamical Systems, and Bifurcations of Vector Fields, Springer Verlag, New York, 1983.

16. Bergé, P.. Pomeau, Y. and Vidal, C., Order within Chaos, John Wiley and Sons, New York, 1984. 
17. McDonough, J.M., Yang, Y. and Hylin, E.C., in Proceedings of First Asian Computational Fluid Dynamics Conference, (Hui et al. Eds.), Vol. 2, Hong Kong University of Science and Technology, 1995, pp. 747-752.

\section{Figure Captions}

Fig. 1: Schematic of the experimental system.

Fig. 2: Experimental data at different flame heights: (a) Time series for the intensity of scattered laser light at $30^{\circ}$; (b) Power spectra density vs. frequency, and (c) the Delay map.

Fig. 3: Power spectra density vs. frequency at two different heights: Based on (a) Averaged data; (b) Unaveraged data.

Fig. 4: Predictions from the model (compare with the data at different flame heights, see Fig. 2): (a) Time series; (b) Power spectra density vs. frequency, and (c) the Delay map. 


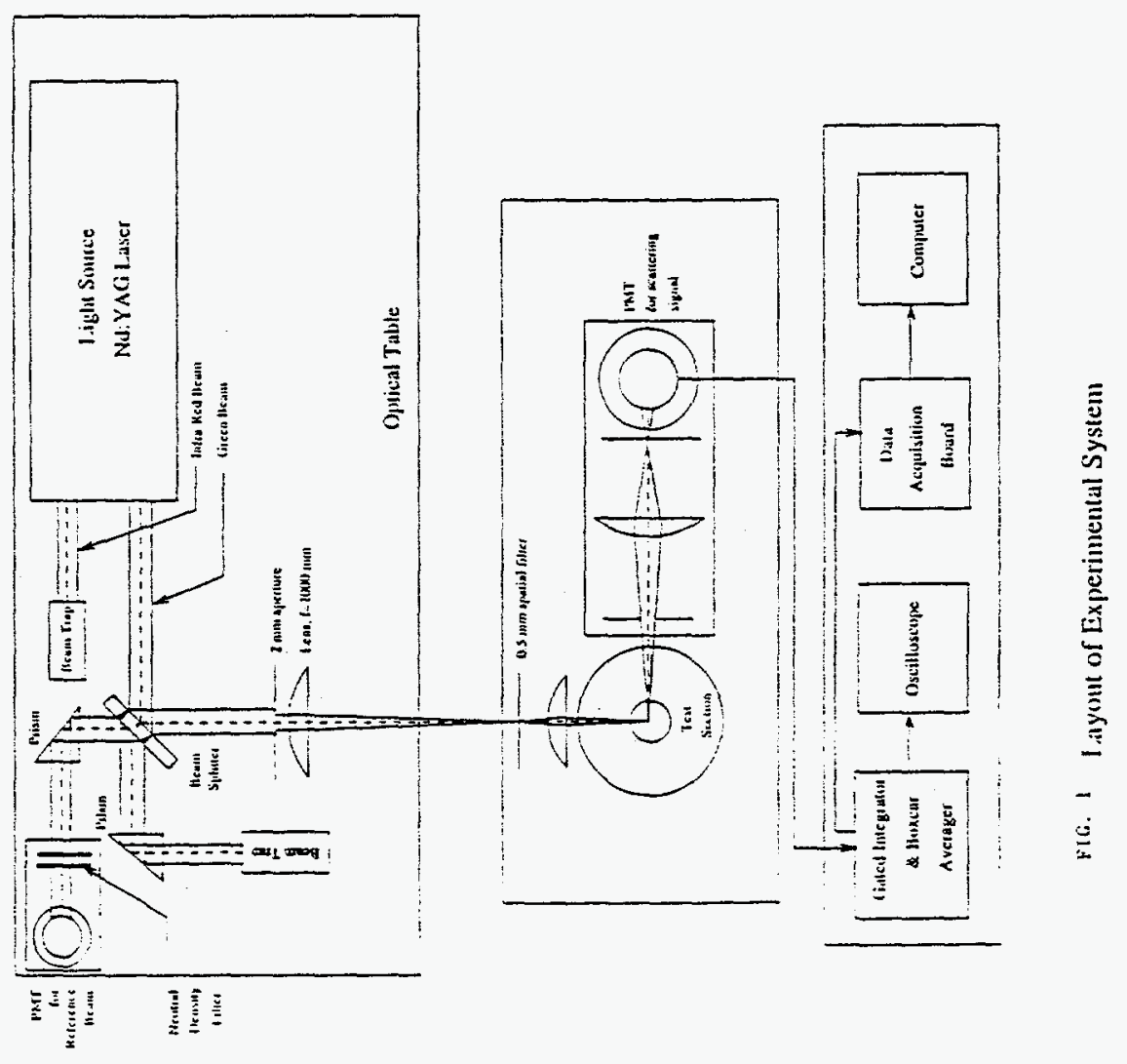




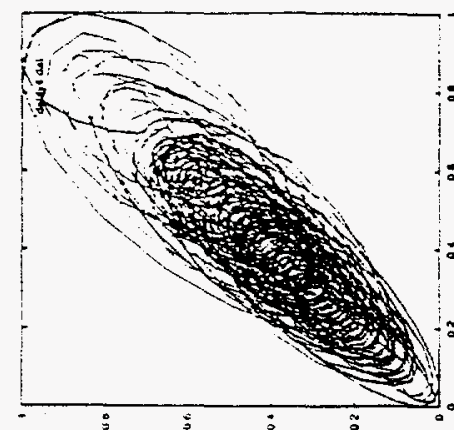

(a) ${ }^{\wedge}$

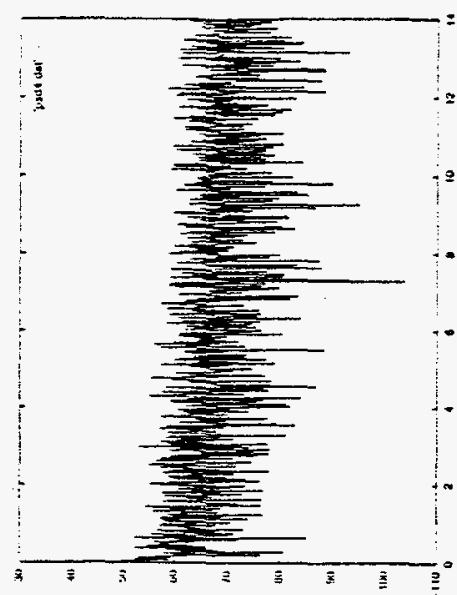

qp 'jamod

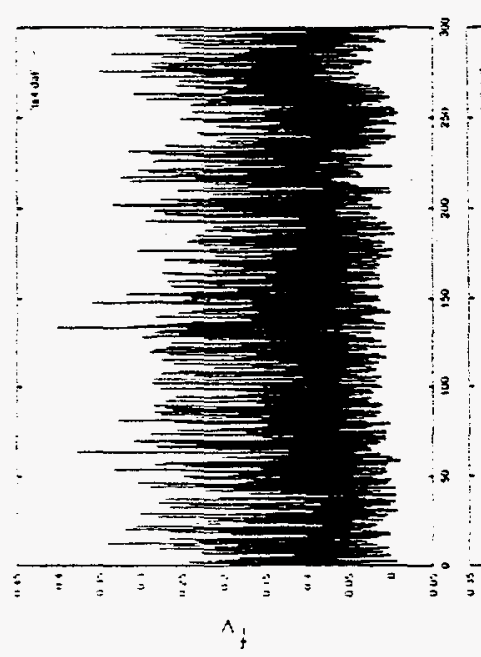

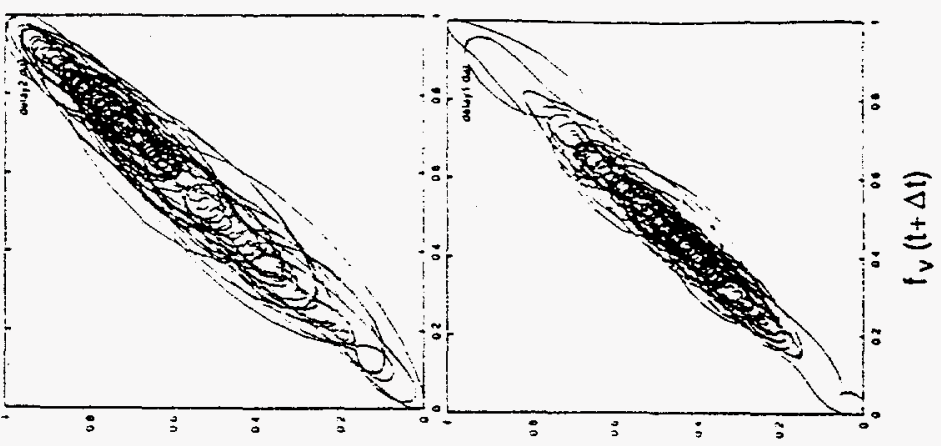

(7) $\wedge^{\wedge}$

(b) ${ }^{\wedge}+$

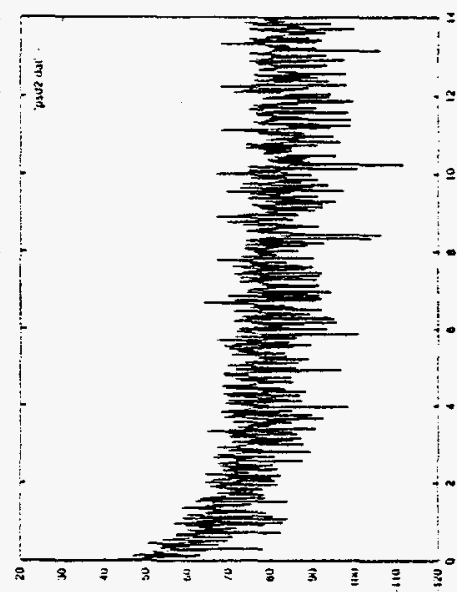

ap 'semod
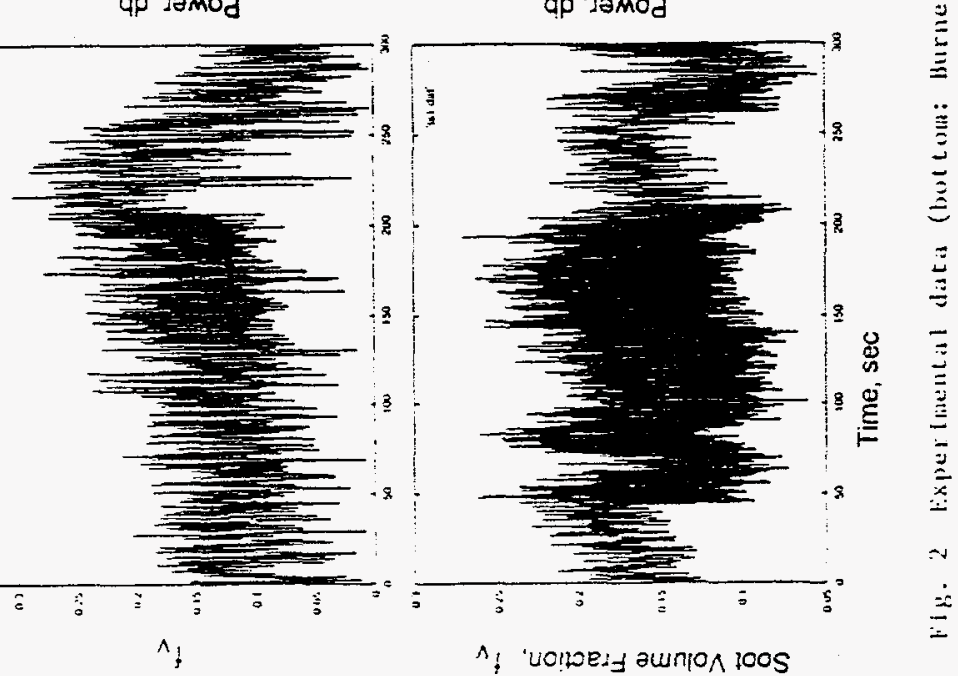
Fig. 3

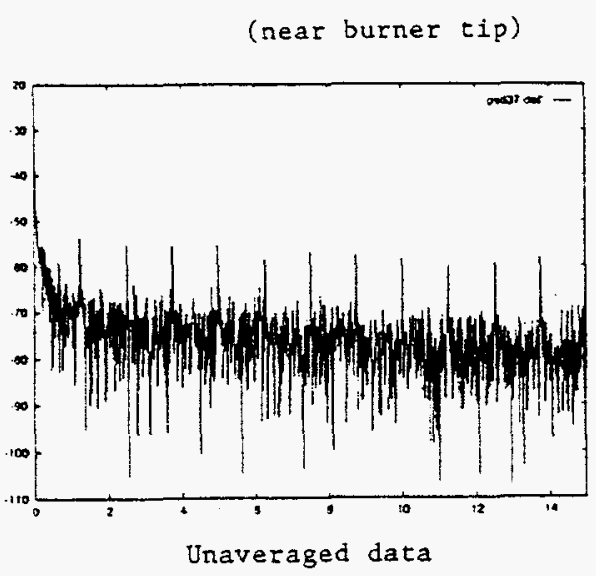

(higher flase location)
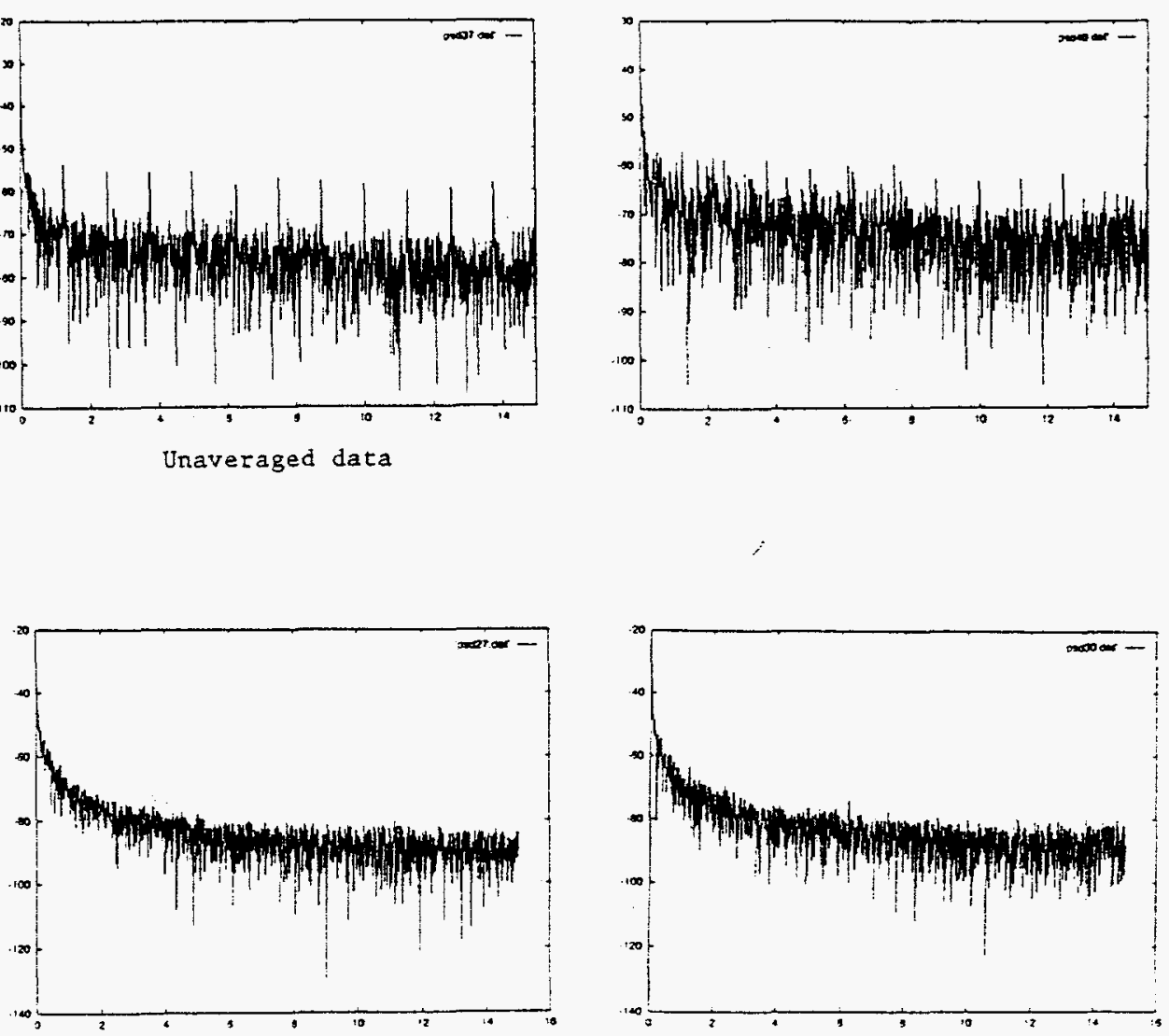

Averaged data 

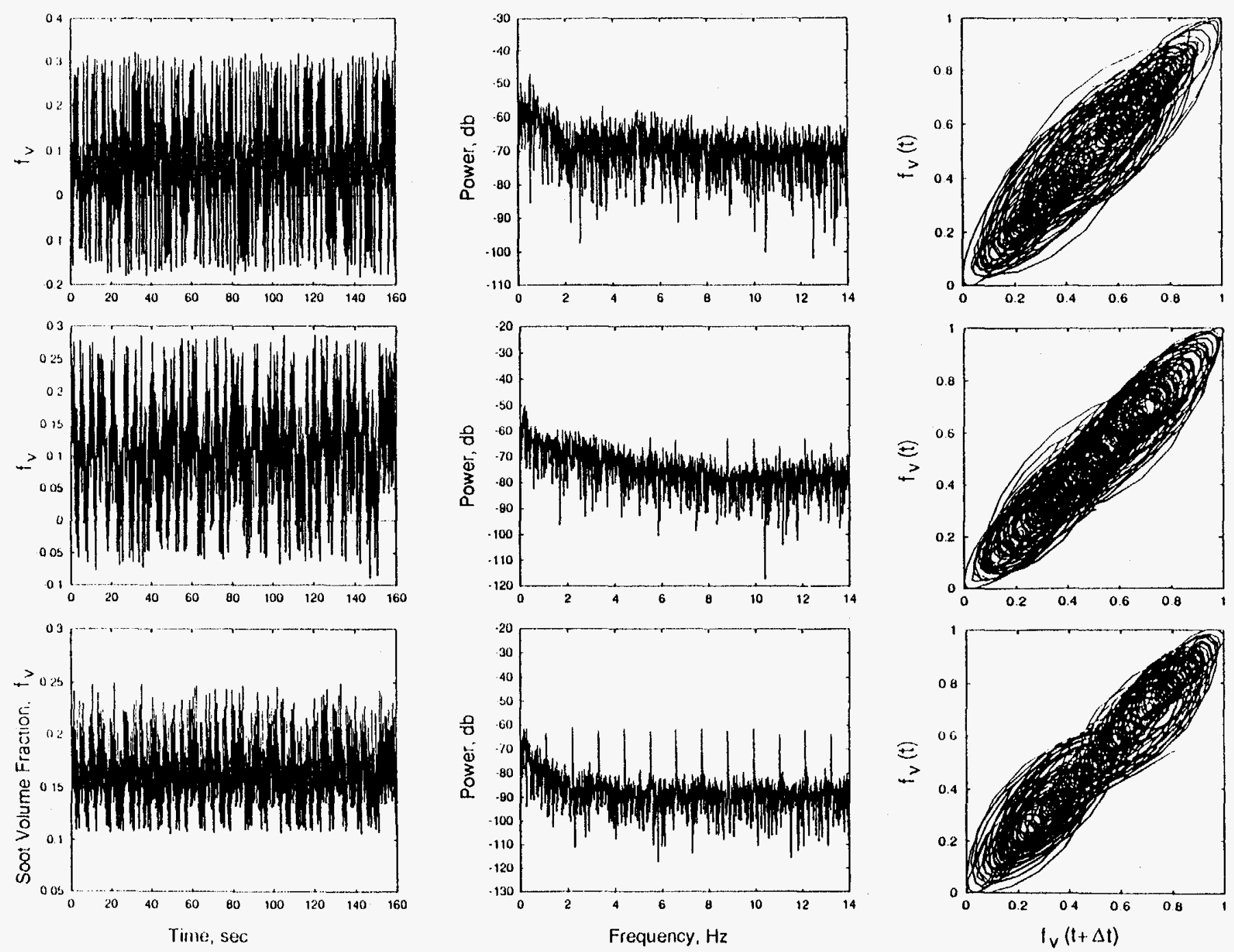

fig. 4 Model predtctions (see Fig. 2 for comparlsons with the expertinental data) 